Case report of hereditary hemorrhagic telangiectasia and primary hyperparathyroidism.

H. Eid, S. Abdul

Introduction:

Epsom hospital, London

The diagnosis of hereditary hemorrhagic telangiectasia (HHT) is definite if 3 of the following criteria are present, possible or suspected if 2 are present and unlikely if fewer than 2 are present:

- Epistaxis. - Telangiectasias - Visceral lesions: gastrointestinal, pulmonary, hepatic, cerebral and spinal - Family history: a first-degree relative with $\mathrm{HHT}$.

\title{
Case presentation:
}

She is 81 years lady, well-known case of HHT. She was found to have hypercalcemia on a routine checkup. Her hypercalcemia was proved to be caused by primary hyperparathyroidism. Patient's blood tests showed hypercalcemia (serum levels of corrected calcium was $2.8 \mathrm{mmol} / \mathrm{L}$ ), hypophosphatemia (phosphorus of $0.75 \mathrm{mmol} / \mathrm{L}$ ), high levels of parathyroid hormone (16 pmol/L) and hypercalciuria. However, she did not have any symptoms of hypercalcemia. Total proteins and albumin levels were normal. Her vitamin $D$, thyroid hormones and other electrolytes were also normal. A neck ultrasound was performed, showing no notable pathologies .

\section{Conclusion and discussion:}

Here we present a case of primary hyperparathyroidism in a patient of HHT. No definite association between HHT and endocrinal disorder was confirmed before. However, a case report described the occurrence of hypoparathyroidism and HHT (1). Another case of Hashimoto thyroiditis and HHT was reported in 2006 (2).

\section{References:}

1-. Cesareo et al. (2011). A Rare Case of Hypoparathyroidism Associated to Rendu-Osler-Weber. ACTA ENDOCRINOLOGICA January 2011, 7(2):267-272.

2- Sabuncu et al. (2006). Hashimoto Thyroiditis in a Patient With Hereditary Hemorrhagic Telangiectasia. The Endocrinologist. 16(1). 2-4. 